Ökologische Bilanzierung städtischer Mobilität

\section{Alte Annahmen bestätigt}

\section{Gelingt die ökologische Bilanzierung städtischer Mobilität - angelehnt an die Ökobilanzen des produzierenden Gewerbes - alleine anhand von Fluß- und Infrastrukturdaten? Besonders im Verkehrsverhalten zeigt sich, daß soziale und städteräumliche Aspekte in eine entsprechende Bilanzierung aufgenommen werden müssen.}

$O^{t}$ Von Katbrin Ankele und Gösta Jancke tädtische Mobilität macht einen nicht unerheblichen Anteil der Stoff- und Energieströme innerhalb einer Stadt aus. Diese Stoffströme sind mit ökologischen und sozialen Auswirkungen verknüpft, die in der Regel in der kommunalen Planung und Umweltpolitik ungenügend zur Kenntnis genommen und berücksichtigt werden. Die ökologische Bilanzierung städtischer Mobilität verfolgt daher das Ziel, auf der Basis der Analyse von Stoff- und Energieströmen Aussagen über deren Auswirkungen treffen zu können. Darüber hinaus werden Anforderungen an eine ökologisch verträgliche Mobilität in Stadtregionen formuliert, unter Berïcksichtigung sozialer Faktoren. Dabei wird über den „klassischen“ Ansatz der Verknüpfung von Fahrleistungen mit Luftemissionen hinausgegangen und die Infrastruktur, die zur Verfïgung gestellt wird, einbezogen. Damit soll ein vollständigeres und aussagefähigeres Bild der Umweltwirkungen verschiedener Verkehrssysteme gezeichnet werden, ferner gilt es. Schwachstellen innerhalb der jeweiligen Systeme erkennbar zu machen. Und nicht zuletzt sollen Optiermierungspunkte aufgezeigt werden. Aufbauend auf der Methode der Ökobilanzierung von Produkten und Dienstleistungen werden zunächst die Flußund Infrastrukturdaten verschiedener Verkehrssysteme ermittelt. Im Anschluß daran werden diese mit der Abschätzung ihrer ökologischen Wirkungen verknüpft und zuletzt einem ökologischen Anforderungsprofil gegenübergestellt.

Die Methode der ökologischen Bilanzierung städtischer Mobilität wird im Rahmen des Verbundprojektes „Ökologisch verträgliche Mobilität in Stadtregionen" im Förderschwerpunkt Stadtökologie des Bundesministeriums für Bildung, Wissenschaft, Forschung und Technologie (BMBF) entwickelt und an den beiden Modellstädten Halle und Bremen erprobt. Nachfolgend werden exemplarisch einige Aussagen und Tendenzen aus Bremen dargestellt und kommentiert. Es handelt sich dabei sowohl um Emissionsbelastungen durch Fahrleistungen als auch um Materialbedarf und Straßenschädigung.

\section{Sachbilanz und Kennzahlen}

Für die Sachbilanz wurden keine Daten eigens erhoben, sondern ausschließlich auf Zahlen aus den städtischen Behörden und Betrieben, von Herstellern betreffender Produkte sowie Literatur zurückgegriffen. Auf diese Weise sollen die Stoff- und Energieströme, die durch Mobilität in den Modellstädten verursacht werden, so vollständig wie möglich erfaßt werden: Neben dem Kraftstoff- und Stromverbrauch zum Betrieb der Verkehrsmittel und den resultierenden Emissionen wurden Daten zu den Quantitäten und den Hauptbestandteilen der Infrastruktur abgefragt, wie z.B. Straßenbeläge, Fahrzeuge, Gleiskörper, Beleuchtungskörper, Signalanlagen, Straßenschilder, Brücken, Betriebsgebäude. Auf der Grundlage des Materialinputs wird zudem die für die Erstellung aufgewandte Energie berechnet.

\section{Emissionen und Materialeinsatz}

Die nachfolgend im Vergleich der drei Personentransportmittel Pkw, Linienbus und Straßenbahn dargestellten Emissionswerte wurden für

Transportleistung in Millionen Personenkilometer [Mio. Pkm] und ausgewählte Emissionen [g/Pkm] von Personenkraftwagen, Linienbussen und Straßenbahnen in Bremen 1990

\begin{tabular}{lccc|}
\hline & Pkw & Linienbus & Straßenbahn \\
\hline Iransportleistung & 2.724 & 250 & 231 \\
\hline Kohlenwosserstoffe & 0,76 & 0,15 & 0,20 \\
\hline Kohlenmonoxid & 6,11 & 0,56 & 0,06 \\
\hline Stickoxide & 1,39 & 1,27 & 0,09 \\
\hline Kohlendioxid & 121,38 & 79,16 & 78,75 \\
\hline Partikel & 0,0096 & 0,0613 & 0,0091 \\
\hline Benzol & 0,0424 & 0,0029 & keine Angaben \\
\hline Toluol & 0,0809 & 0,0012 & keine Angaben \\
\hline Dieselluo & 0,0058 & 0,0245 & keine Angaben \\
\hline
\end{tabular}

Quelle: IÖW 1996 haltet die nach Meinung der Stadt Bremen für eine Verkehrsumlegung relevanten Strecken. Auch wenn dieses Kriterium quantitativ nicht faßbar ist, kann doch davon ausgegangen werden, daß dieses Modell, das rund 35 Prozent der Gesamtlänge des Bremer Verkehrswegenetzes abbildet, die wesentlichen $\mathrm{Kfz}$-Ströme berïcksichtigt.

Aus der Vielzahl der verkehrsbedingten Emissionen werden im folgenden einige bedeutende klimarelevante, karzinogene oder toxische Abgaskomponenten vergleichend für die Verkehrsmittel Pkw, Linienbus und Straßenbahn dargestellt (siehe Tabelle). Die Tabelle zeigt, daß die öffentlichen Verkehrsmittel (ÖV), vor allem die Straßenbahn, pro Personenkilometer (Pkm) bei den meisten Emissionen deutlich besser abschneiden als der Pkw. Eine Ausnahme bilden die Partikel und der Dieselruß, die für Dieselmotoren spezifisch sind. Einem im Vergleich zum Pkw etwa viermal so hohen Dieselrußausstoß der Busse steht allerdings eine um den Faktor 14 (Benzol) bzw. 67 (Toluol) größere Emission besonders gefährlicher Kohlenwasserstoffe gegenüber. Dieselruß und Benzol gelten als krebserregend, Toluol ist neurotoxisch. $\mathrm{Zu}$ beachten ist zudem, daß bei der gegebenen.

Pkw und Bus auf der Grundlage der Verkehrserhebungen der Stadt Bremen im Rahmen der integrativen Verkehrsplanung (1), der Zulassungszahlen des Kraftfahrtbundesamtes sowie des Handbuchs der Emissionsfaktoren des Umweltbundesamtes (2) berechnet. Für die Straßenbahn wirde ein deutscher Strommix entsprechend GEMIS 2.1 (3) zugrundegelegt. Das digitalisierte Straßennetz, das für die integrative Verkehrsplanung erstellt worden ist und für das die Verkehrsbelastung bekannt ist, bein-

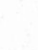

.


Transportleistung von mehr als 2,7 Milliarden Pkm in Bremen im Jahr 1990 mehrere hundert Tonnen der gefährlichen Kohlenwasserstoffe (Benzol, Toluol und Xylol) allein durch Pkw emittiert werden. Ein Großteil davon fällt in kleineren Anwohnerstraßen an.

Auch in Bezug auf den Materialeinsatz für die Bereitstellung der Fahrzeuge und der Fahrwege (Grafik) verhält sich der ÖV günstiger als der motorisierte Individualverkehr (MIV). Unter Zugrundelegung geeigneter Annahmen über Abschreibungszeiten ergibt sich, daß für jeden Pkm im Pkw mehr als acht mal soviel Material wie für die Straßenbahn und mehr als elf mal soviel wie für den Linienbus aufgewendet wurde. Auch wenn die Aussagefähigkeit dieses Wertes, der alle Substanzen unabhängig von ihren spezifischen Eigenheiten zum gesamten Materialeinsatz aufsummiert enthält, für eine Dienstleistung (hier Personentransport) nicht überbewertet werden sollte, so deutet doch auch dieser Parameter auf eine größere ökologische Verträglichkeit des ÖV gegenüber dem MIV hin.

\section{- Straßenschäden}

Ein anderes Bild ergibt sich, betrachtet man die Beiträge von Pkw und Bus zur Beschädigung der Straßensubstanz. In diese Betrachtung müssen die Lkw mit einbezogen werden, während Straßenbahnen unberïcksichtigt bleiben. Grundlagen der Berechnungen bilden das Gewicht der Fahrzeuge, das nach der zur Zeit üblicherweise verwendeten Formel der American Association of State Highway Officials (AASHO) über die vierte Potenz der Achslast eingeht, sowie die geleisteten Fahrzeugkilometer in Bremen 1990.

Berechnungen zeigen, daß aufgrund der großen Bedeutung des Gewichts die relativ leichten Pkw trotz ihres hohen Anteils am Verkehrsaufkommen nur unwesentlich zu den Straßenschäden beitragen, während die schweren Fahrzeuge Bus und Lkw dafür hauptverantwortlich sind. Aufgrund der unvollständigen Datenbasis ist die Umrechnung der Verkehrsanteile auf die Achsenlast aller Lkw-Nutzlastklassen sehr schwierig. Die dafür notwendigen Informationen (genaue Zuordnung der Güterverkehrsmengen zu den Lkw. Nutzlastklassen, Kenntnis über die Anzahl der Achsen der Fahrzeuge, Zusammenhang zwischen Fahrleistung und Gewicht der Ladung) müssen für den innerstädtischen Bereich berechnet werden. Es wird dennoch deutlich, daß auch die Linienbusse nicht unerheblich zur Beschädigung der Straßensubstanz beitragen.

\section{Schlußfolgerungen}

Aus diesen wenigen Beispielen können folgende Schlußfolgerungen gezogen werden:

- Erstens wird auch bei Einbeziehung der Infrastruktur und des Materialbedarfs die bisherige Annahme bestätigt, daß im Personennahverkehr Mobilität mit dem Bus und vor allem mit der Straßenbahn deutlich ökologisch verträglicher ist als mit dem Pkw.

- Zweitens wird jedoch deutlich, daß allein durch eine Veränderung des Modal Split keine ausreichende ökologische Entlastung erreicht werden kann, da auch die Bereitstellung des ÖV mit erheblichen Umweltbelastungen verbunden ist. Es muß daher bei der Neuoder Umgestaltung der Rahmenbedingungen für Mobilität in Stadtregionen über Vermeidungsstrategien nachgedacht und Abschied genommen werden von der Vorstellung, motorisierten Verkehr bei gleichbleibendem Aufkommen durch Modal-Split-Veränderungen umweltverträglich realisieren zu können.

\section{Kontext sozialer und stadträumlicher Bedingungen}

Das Ziel einer umfassenden Ökobilanz ist es wie eingangs erwähnt -, auf der Basis der dargestellten Daten und Berechnungen Aussagen über die ökologischen Auswirkungen zu treffen und dabei nicht allein auf bestimmte Luftschadstoffe abzuheben. Insbesondere sollen neben ökologischen auch soziale Kriterien einbezogen werden, um diesem umfassenderen Anspruch gerecht zu werden.

In der „Ökobilanz von Transportvorgängen in Stadtregionen" werden daher zusätzlich zu den oben beschriebenen quantitativen Aussagen Anforderungsprofile entwickelt: zum einen an ökologisch verträgliche Mobilität, zum anderen in bezug auf die Datenbasis, die Städten zur Verfügung stehen müßte, um Mobili-tät annähernd vollständig und damit aussagekräftig bilanzieren zu können. Das heißt, daß neben der Bewertung der vorhandenen Informationen - des Ist-Zustandes - Anforderungen beschrieben werden, die ökologisch verträgliche Mobilität auch im Kontext sozialer und stadträumlicher Bedingungen beschreiben. Dazu werden handhabbare Kriterien und Standards als Zielvorgaben für ökologisch verträgliche Mobilität entwickelt. Die ökologischen Kriterien beziehen sich sowohl auf Einzelsubstanzen wie Kohlendioxid, Benzol etc. als auch auf Umweltwirkungen wie

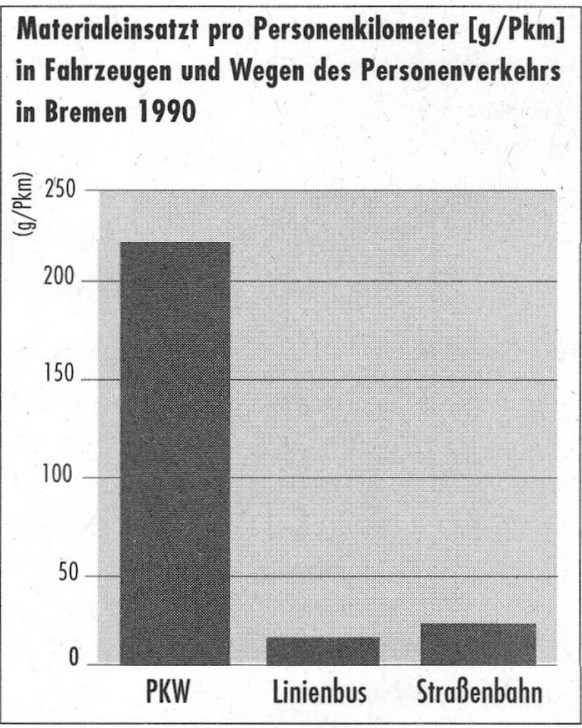

Treibhauseffekt, die Versauerung etc. Die sozialen Anforderungen umfassen unter anderem Aufenthaltsqualität (auf Straßen und Plätzen), Sicherheit im Straßenraum oder Wegequalität für Fußgänger und Radfahrer. Innerhalb dieser Kriterien werden Standards formuliert, die sich sowohl an Grenzwerten orientieren als auch an Handlungs- und Qualitätszielen. Es bleibt zu klären, ob diese Standards spezifisch für Zielgruppen (Kinder, alte Menschen, Familien, etc.) und Gebietstypen (Wohngebiet, Industriegebiet, Innenstadt) entwickelt werden können. Ausgehend von den Kriterien und Standards wird im Anschluß untersucht, welche Handlungsstratęgien und Mobilitätsvarianten zu deren Erfiillung führen.

\section{Anmerkungen}

1) Integrative Verkehrsplanung Bremen. Untersuchung im Auftrag des Senators für das Bauwesen, Bremen. PROGNOS/IVV, Basel/Aachen, 1992

2) Emissionsfaktoren für verschiedene Fahrzeugschichten, Straßenkategorien, Verkehrszustände und Bezugsjahre (II). 3. Zwischenbericht. UBA F+E-Vorhaben 10506 044, FIGE, Herzogenrath, 1995

3) Gesamt-Emissions-Modell Integrierter Systeme (GEMIS). Version 2.1. ÖKO-INSTITUT,

Darmstadt/Freiburg/Berlin/Kassel, 1994

\section{Der Autorinnen}

Kathrin Ankele, Dipl.-Biol., und Dr. Gösta Jancke, Dipl.-Ełol., sind tätig im Forschungsfeld „Ökologische Untenehmenspolitik“ des IÖW, Berlin 
(c) 20I0 Authors; licensee IÖW and oekom verlag. This is an article distributed under the terms of the Creative Commons Attribution Non-Commercial No Derivates License (http://creativecommons.org/licenses/by-nc-nd/3.o/), which permits unrestricted use, distribution, and reproduction in any medium, provided the original work is properly cited. 